\title{
High buprenorphine-related mortality is persistent in Finland
}

\author{
Kriikku, Pirkko
}

2018-10

Kriikku , P , Hakkinen , M \& Ojanpera , I 2018 , ' High buprenorphine-related mortality is persistent in Finland ' , Forensic Science International , vol. 291 , pp. 76-82 . https://doi.org/10.1016/j.forsciint.2018.0

http://hdl.handle.net/10138/306898

https://doi.org/10.1016/j.forsciint.2018.08.010

unspecified

publishedVersion

Downloaded from Helda, University of Helsinki institutional repository.

This is an electronic reprint of the original article.

This reprint may differ from the original in pagination and typographic detail.

Please cite the original version. 


\title{
High buprenorphine-related mortality is persistent in Finland
}

\author{
Pirkko Kriikku, a,b,*, Margareeta Häkkinen ${ }^{c}$, Ilkka Ojanperäa,b \\ ${ }^{a}$ Forensic Toxicology Unit, National Institute for Health and Welfare, P.O.Box 30, 00271 Helsinki, Finland \\ ${ }^{\mathrm{b}}$ Department of Forensic Medicine, University of Helsinki, Finland \\ ${ }^{\mathrm{c}}$ A-Clinic Foundation, Addiction Hospital, Finland
}

\section{A R T I C L E I N F O}

\section{Article history:}

Received 5 June 2018

Received in revised form 6 August 2018

Accepted 7 August 2018

Available online 17 August 2018

\section{Keywords:}

Buprenorphine

Fatal poisoning

Overdose

Drug abuse

Opioid maintenance treatment

\begin{abstract}
A B S T R A C T
Sublingual buprenorphine is used in opioid maintenance treatment but buprenorphine is also widely abused and causes fatal poisonings. The aim of this study was to investigate buprenorphine-positive fatalities in order to gain novel information on the magnitude and nature of buprenorphine abuse.

All post-mortem toxicology cases positive for urinary buprenorphine, including fatal poisonings caused by buprenorphine and fatalities in which the cause of death was unrelated to buprenorphine, in the five year period of 2010-2014 in Finland were characterized according to urine buprenorphine and naloxone concentrations $(n=775)$. Urine concentrations were used to assess which buprenorphine preparation had been used; mono-buprenorphine or a buprenorphine-naloxone combination, and whether they had been administered parenterally.

In at least $28.8 \%$ of the buprenorphine-positive cases the drug had been administered parenterally. The majority of the parenteral users (68.6\%) had taken mono-buprenorphine. Fatal poisoning was significantly more common among the identified parenteral users (65.5\%) than among other users of buprenorphine products (45.3\%). The proportion of buprenorphine-related poisoning was similar in identified parenteral users of mono-buprenorphine (68.6\%) and buprenorphine-naloxone (64.1\%). In nearly all of the fatal poisoningss the deceased had used other drugs and/or alcohol along with buprenorphine (98.7\%). The median age of the deceased increased significantly over the study period, from 32 to 38 years.

Our results show that there is ongoing parenteral abuse of both mono-buprenorphine and buprenorphine-naloxone combination. Parenteral users of buprenorphine put themselves into a great risk of fatal poisoning or other accidental injury death which is further exacerbated by the frequent polydrug use.
\end{abstract}

(c) 2018 Elsevier B.V. All rights reserved.

\section{Introduction}

Opioids cause more fatal drug poisonings in the European Nordic countries than any other pharmacological drug class, and, in Finland buprenorphine is the most commonly abused opioid [1]. Buprenorphine is a semi-synthetic partial agonist at the $\mu$-opioid receptor with antagonist activity at the $\kappa$-opioid receptor. Sublingual buprenorphine (Subutex ${ }^{\circledR}$, BPN) has been used in the treatment of opioid dependence in Finland since 2000 and has been shown to be safe in opioid maintenance treatment (OMT) [2].

Buprenorphine has a special history in Finland: two general practitioners in Helsinki introduced the use of buprenorphine for the treatment of opioid addicts in the 1990s [3]. However, the Finnish authorities considered their methods and integrity to be questionable and later one of the physicians was found guilty of

\footnotetext{
${ }^{*}$ Corresponding author at: Forensic Toxicology Unit, National Institute for Health and Welfare, P.O.Box 30, 00271 Helsinki, Finland.

E-mail address: pirkko.kriikku@thl.fi (P. Kriikku).
}

smuggling and selling buprenorphine tablets to his patients. This early introduction of buprenorphine likely influenced the evolution of the Finnish recreational drug scene so that currently there is frequent parenteral abuse (injecting or snorting the drug) of buprenorphine while parenteral use of heroin is less common [1].

BPN was first started in OMT in Finland in 1997 [4]. During the 2000s, the use of OMT increased and was implemented by health centres [3]. A combination of buprenorphine and naloxone for sublingual administration (Suboxone ${ }^{\circledR}$, BNX) became available at the end of 2003. Several treatment centres switched their patients rapidly from BPN to BNX because the combination sublingual product appears to be less likely to be solubilized and abused parenterally, although this does still occur [5]. With the exception of some programs that still treat pregnant women with BPN, since 2009, BNX has been the sole buprenorphine-containing product used to treat OMT patients in Finland. In 2015, 63\% of the Finnish OMT patients were taking buprenorphine or buprenorphine containing combinations and 37\% were taking methadone [6]. 
Despite buprenorphine having a favourable safety profile in medically supervised use compared to, e.g., methadone [7], results from several French case series suggested that intravenous abuse of buprenorphine, when associated with other psychotropic drugs, is a major risk factor for fatal poisoning [8-10]. A later populationbased Finnish study confirmed that benzodiazepines and alcohol are associated with cases of fatal buprenorphine poisoning [11]. In addition, animal studies have shown that buprenorphine can cause respiratory depression, especially when administered together with alcohol [12] or other central nervous system depressants such as benzodiazepines $[13,14]$, but the interaction is complex and not yet thoroughly understood [12,15-17]. In any case, fatal poisoning by buprenorphine alone without other central nervous system depressants seems to be uncommon [8-13]. In Finland, opioid users generally take multiple drugs of abuse from different pharmacological classes and the majority of fatal poisonings of opioid dependent individuals are poly-drug poisonings [1].

Surveys have indicated that intravenous injection is the most common route of administration for individuals abusing buprenorphine [18-20]. Since the introduction of BNX it has become evident that both sublingual formulations, BPN and BNX, are abused intravenously, although the latter to a lesser extent [21-23]. In addition, BPN and BNX are also subject to considerable diversion and trafficking [24-26]. Until recently, it has not been possible to differentiate between BPN and BNX abuse by laboratory analysis. Our earlier study, based on post-mortem toxicology investigation in Finland, was the first laboratory-based study to assess whether these drugs had been administered parenterally, and the study demonstrated the toxicity associated with parenteral abuse of BPN and BNX [27].

In this study, our objective was to investigate BPN and BNX abuse and fatal poisonings in a larger study population and look for trends over time, using our previously published methodology to determine which buprenorphine product had been abused [27].

\section{Material and methods}

All deaths in the medicolegal investigations involving buprenorphine in the five-year period between 2010 and 2014 were examined in this study.

In Finland, all sudden and unexpected deaths are required by law to undergo a medicolegal investigation, initiated by the police and conducted by a forensic pathologist. As a result, in nearly $20 \%$ of all deaths a medicolegal investigation is performed with an even higher percentage in younger individuals.

In most medicolegal cases during the study period, the forensic pathologist had requested a post-mortem toxicological examination. This included the screening of post-mortem urine by ultrahigh performance liquid chromatography coupled with highresolution time-of-flight mass spectrometry (LC-TOFMS) [28,29], screening and quantification of post-mortem blood by gas chromatographic (GC) methods [30], and additional confirmation analyses of drugs of abuse by LC-MS/MS and GC-MS methods. The toxicological screening covered more than 700 drugs and poisons, including opioids, amphetamines, cannabinoids and benzodiazepines.
Buprenorphine, its major active metabolite norbuprenorphine, and naloxone were screened by LC-TOFMS in post-mortem urine after treatment with $\beta$-glucuronidase to release free drug from glucuronides [28,29]. Quantification was performed by an LC-MS/ MS method described elsewhere [31]. The lower limit of quantification (LLOQ) in urine for each of the studied substance was $0.10 \mu \mathrm{g} / \mathrm{L}$. Ethanol was analysed in post-mortem blood by headspace gas chromatography and a laboratory cut-off of $0.2 \mathrm{~g} / \mathrm{kg}$ was applied.

In addition to the analytical results, demographic characteristics and information from the death certificate issued by forensic pathologist were collected. Mortality rates were calculated as the number of deaths per 100,000 inhabitants in the 15-64-years-old age group.

\subsection{Data selection and subgroups}

Medicolegal cases, in which the urine sample was positive for buprenorphine, were divided into three groups according to the urine buprenorphine and naloxone concentrations, using the criteria previously developed by analysing a series of urine samples from living individuals in an OMT program [31]: "Parenteral BNX", "Parenteral BPN" and "Other BNX and BPN". These criteria enable post-mortem cases to be categorized according to the route of administration and the preparation used [27].

The grouping criteria in our study have been defined so that the parenteral user groups should not include many individuals that have taken the drug sublingually. However, although the group "other BNX and BPN" contains mostly sublingual users in the study, it is likely to also contain cases of parenteral users.

The cause and manner of death were defined by the forensic pathologist based on autopsy findings, background information and laboratory results including forensic toxicology, biochemistry and DNA analysis, when requested. In this study, the cases were categorized into the following manners of death: accident, illness, suicide and other (incl. homicide, occupational disease, undetermined intent and traffic death).

\subsection{Statistical evaluation}

All statistical analyses were carried out using IBM SPSS software (version 22.0). As the frequency distributions of the urine concentrations and the age of the deceased were skewed, medians were used to characterize the data.

\subsection{Ethical issues}

The study was conducted in accordance with all applicable local and international laws and regulations. For this type of study, formal consent is not required.

\section{Results}

In the five year period between 2010 and 2014, post-mortem toxicology was applied to 33,420 cases. The total number of cases that met the inclusion criteria of this study (urine sample positive

Table 1



\begin{tabular}{|c|c|c|c|c|c|c|c|c|c|c|c|c|}
\hline & \multicolumn{4}{|c|}{ Parenteral BNX } & \multicolumn{4}{|c|}{ Parenteral BPN } & \multicolumn{4}{|c|}{ Other BNX or BPN } \\
\hline & $\mathrm{N}$ & Median & $(95 \% \mathrm{CI})$ & Range & $\mathrm{N}$ & Median & $(95 \% \mathrm{CI})$ & Range & $\mathrm{N}$ & Median & $(95 \% \mathrm{CI})$ & Range \\
\hline Buprenorphine & 70 & 180 & $140-220$ & $21-1300$ & 153 & 110 & $96-120$ & $50-1500$ & 552 & 15 & $12-17$ & $1-680$ \\
\hline Norbuprenorphine & 69 & 64 & $29-100$ & $1.2-1000$ & 149 & 51 & $31-71$ & $1.2-520$ & 494 & 12 & $10-14$ & $1-700$ \\
\hline Naloxone & 70 & 220 & $170-260$ & $100-2600$ & 0 & & & & 171 & 20 & $15-25$ & $1-98$ \\
\hline
\end{tabular}


for buprenorphine) was 775 , which represents $2.3 \%$ of all cases in that time period. Of the urine samples in the studied 775 cases, 712 (91.9\%) contained norbuprenorphine and 241 (31.1\%) contained naloxone. The mean and median concentrations of buprenorphine, norbuprenorphine and naloxone in different user groups are given in Table 1.

\subsection{User groups}

Of the 775 cases selected for the study, in 223 (28.8\%) the measured urine concentrations indicated recent parenteral administration of buprenorphine. In 70 of these $(9.0 \%$ of all buprenorphine positive cases), naloxone was detected together with buprenorphine indicating recent parenteral administration of BNX. In 153 (19.7\% of all buprenorphine positive cases) of the parenteral buprenorphine cases no naloxone was detected indicating recent parenteral administration of BPN or another mono-buprenorphine preparation. In the remaining 552 (71.2\%) cases the measured urine concentrations were below the threshold that would identify them as parenteral users, although some could be parenteral users whose urine levels had failed to achieve or had fallen below the thresholds.

No significant changes in the total number of buprenorphinepositive cases in the five consecutive years were observed. Also, the proportion of cases in each group remained relatively stable over the study period (Table 2 ).

The median (range) age of the deceased in the study population was 33 (18-98) years with $89.9 \%$ being aged 15-64. Further breakdown of the yearly numbers is given in Table 2 .

The median age of the deceased was significantly lower in the parenteral BNX and BPN groups when compared to the group "other BNX or BPN" ( $\mathrm{P}=0.001$ and $\mathrm{P}<0.001$, respectively). However, no significant difference was detected between the two groups of parenteral users. The age of the deceased increased significantly over the study period $(\mathrm{p}<0.001)$ (Fig. 1$)$.

Of the total 775 buprenorphine-positive cases, $83 \%$ were males, and the proportion of males was over $80 \%$ in each of the three user groups. The gender distribution in different age groups and in the three user groups is illustrated in Fig. 2. Differences were observed in the proportion of males in different age groups, especially among the oldest individuals: in the age group 60 years and above, the precentage of males was $54.5 \%(\mathrm{p}<0.001)$.

\subsection{Fatal poisonings and concomitant use of other drugs}

Of the 775 studied cases, 396 (51.1\%) were fatal poisonings in which buprenorphine was implicated as the cause of death by the

\section{Table 2}

Number of deceased, and median age and gender in each user group during 2010-2014.

\begin{tabular}{lllllll}
\hline & 2010 & 2011 & 2012 & 2013 & 2014 & TOTAL \\
\hline Parenteral BNX & & & & & & \\
$\quad$ N & 23 & 13 & 13 & 11 & 10 & 70 \\
$\quad$ Median age (y) & 28 & 32 & 28 & 36 & 35 & 31 \\
$\quad$ Males (\%) & 83 & 92 & 92 & 100 & 80 & 89 \\
Parenteral BPN & & & & & & \\
$\quad$ N & 35 & 31 & 23 & 28 & 36 & 153 \\
$\quad$ Median age (y) & 29 & 30 & 30 & 29 & 37 & 31 \\
$\quad$ Males (\%) & 77 & 87 & 82 & 86 & 92 & 85 \\
Other BNX or BPN & & & & & & \\
$\quad$ N & 101 & 108 & 100 & 123 & 120 & 552 \\
$\quad$ Median age (y) & 33 & 33 & 36 & 37 & 41 & 36 \\
$\quad$ Males (\%) & 84 & 81 & 83 & 80 & 82 & 82 \\
TOTAL & & & & & & \\
$\quad$ N & 159 & 152 & 136 & 162 & 166 & 775 \\
$\quad$ Median age (y) & 32 & 31 & 33 & 35 & 38 & 33 \\
$\quad$ Males (\%) & 82 & 83 & 84 & 82 & 84 & 83 \\
\hline
\end{tabular}

forensic pathologist. Buprenorphine was considered to be the primary finding in 256 of the fatal poisonings (33.0\%) (Table 3.). The proportion of buprenorphine poisonings was significantly higher in the groups "parenteral BNX" (68.6\%) and "parenteral BPN" (64.1\%) than in the group "other BNX or BPN" $(45.3 \%)$ $(\mathrm{p}=0.001$ and $\mathrm{p}<0.001$, respectively).

The yearly number of fatal buprenorphine poisonings decreased significantly during the study period $(\mathrm{p}<0.05)$. Accordingly, the mortality rate calculated per 100,000 Finnish inhabitants between 15 and 64 years of age decreased from 2.7 to 1.9. Of the studied user groups, the decrease in the number of fatal poisonings was significant in all except in the group "parenteral BPN". A further breakdown of the poisoning numbers is given in Table 4 .

There were five fatal poisonings in which no other psychoactive substances, besides buprenorphine, were present in pharmacologically relevant concentrations. A typical example of these five mono-intoxications was a case in which a 28 -year old male was found dead in his apartment lying next to a syringe and, in addition to buprenorphine, only cannabis (at a below pharmacologically relevant level) was detected in post-mortem toxicology.

The blood alcohol concentration (BAC) was above the laboratory cut-off of $0.2 \mathrm{~g} / \mathrm{kg}$ in 281 cases (37.2\%) and above $0.5 \mathrm{~g} / \mathrm{kg}$ in 235 cases $(30.3 \%)$, the highest BAC being $3.4 \mathrm{~g} / \mathrm{kg}$. The mean BAC was $1.33 \mathrm{~g} / \mathrm{kg}$ for all blood alcohol-positive cases, $1.10 \mathrm{~g} / \mathrm{kg}$ for blood alcohol-positive "parenteral BNX" group and $1.20 \mathrm{~g} / \mathrm{kg}$ for blood alcohol-positive "parenteral BPN" group. The percentage of cases with a BAC above the laboratory cut-off of $0.2 \mathrm{~g} / \mathrm{kg}$ was slightly lower in the groups of parenteral users (BNX 30.0\% and BPN 33.3\%) compared to the group "Other BNX and BPN" but the difference was not statistically significant. Among those who died of fatal buprenorphine poisoning, $45.2 \%$ had a BAC over the cut-off of $0.2 \mathrm{~g} /$ $\mathrm{kg}$ with a mean BAC of $1.37 \mathrm{~g} / \mathrm{kg}$.

\subsection{Manner of death}

In the majority of the studied buprenorphine-positive cases the manner of death determined by the forensic pathologist was accident (60.0\%). The proportion of accidental deaths was higher among parenteral users: "Parenteral BNX" (70.0\%) and "Parenteral BPN" (62.1\%) compared to the group "Other BNX and BPN" (57.8\%) but the difference was not statistically significant. Other major manners of death in the study population were illness (21.5\%) and suicide (11.2\%). Further breakdown of the numbers in different user groups is illustrated in Fig. 3.

\section{Discussion}

Based on the laboratory analysis, 223 (28.8\%) of the 775 deceased included in the study could be identified as parenteral users of buprenorphine preparations. This is in line with the results of our previous study in which the proportion was $31.1 \%$ [27]. This number must be considered a minimum since the urine concentrations of some parenteral users may not have reached, or may have fallen below, the threshold levels at the time of death. Such cases could not be distinguished from sublingual users and would not be included in the parenteral groups.

Even though the quantity of BPN used for approved medical purposes in Finland is very much less than that of BNX, most of the identified parenteral users had been using BPN (153) rather than BNX (70). Some mono-buprenorphine preparations are available in Finland for the treatment of severe pain, including for example, Norspan ${ }^{\circledR}$ patches and the tablet preparation Temgesic ${ }^{\circledR}$. These buprenorphine products also possess abuse potential and are likely subject to some degree of diversion, although, because of their relatively low sales numbers, it seems unlikely that they could be a major source of the buprenorphine that is abused. In a recent 


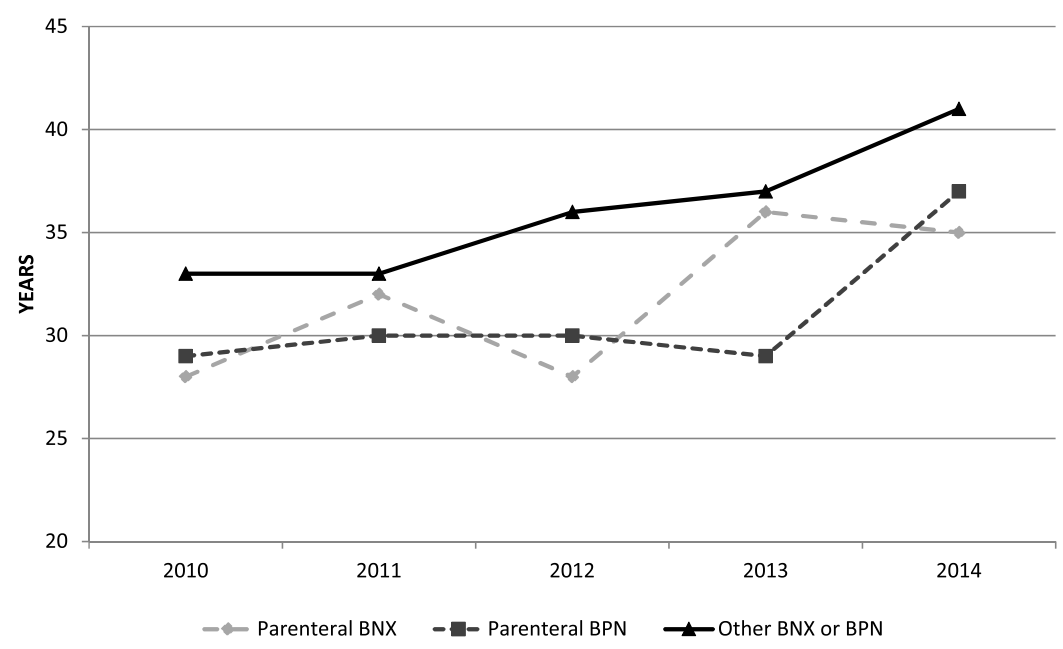

Fig. 1. Median age of deceased in each user group during 2010-2014.



Fig.2. Age and gender distribution of deceased in each user group.

Table 3

Number of fatal buprenorphine poisonings and other causes of death in each user group. Percentages within each group are given in brackets.

\begin{tabular}{|c|c|c|c|c|}
\hline Cause of death & Parenteral BNX & Parenteral BPN & Other BNX or BPN & Total \\
\hline \multicolumn{5}{|l|}{ Fatal poisoning } \\
\hline Buprenorphine implicated & $48(68.6 \%)$ & $98(64.0 \%)$ & $250(45.3 \%)$ & $396(51.1 \%)$ \\
\hline Buprenorphine primary & $41(58.6 \%)$ & $78(51.0 \%)$ & $137(24.8 \%)$ & $256(33.0 \%)$ \\
\hline Other cause of death & $22(31.4 \%)$ & 55 (35.9\%) & $302(54.7 \%)$ & $379(48.9 \%)$ \\
\hline Total & 70 & 153 & 552 & 775 \\
\hline
\end{tabular}

Table 4

Yearly numbers of fatal poisonings, and mortality rates in the general population of all Finnish inhabitants aged between 15 and 64 .

\begin{tabular}{llllll}
\hline Fatal poisonings \\
\hline Buprenorphine implicated & 2010 & 2011 & 2012 & 2013 & 2014 \\
\hline Parenteral BNX & 18 & 11 & 8 & 6 & 5 \\
Parenteral BPN & 21 & 25 & 15 & 18 & 19 \\
Other BNX or BPN & 56 & 60 & 48 & 45 & 41 \\
All buprenorphine poisonings & 95 & 96 & 71 & 69 & 65 \\
Mortality rate (per 100,000) & 2.7 & 2.7 & 2.0 & 2.0 & 1.9 \\
\hline
\end{tabular}

survey among OMT patients in Finland, injecting illegally acquired opioids was significantly more common than injecting one's own OMT medication [32]. Overall, our results, along with information from treatment programs and police sources, suggest that the major source of abused buprenorphine in Finland is unlikely to be diversion from OMT programs or other medical uses and is probably trafficking from abroad.

Buprenorphine was considered to be the primary finding in 256 fatal poisonings but only a few of these were mono-intoxications by buprenorphine. In the groups which include only parenteral users of BPN or BNX, the proportion of fatal poisonings was higher (65.5\%) than in all studied cases (51.1\%) which underlines the hazards of self-injecting buprenorphine without medical supervision.

The proportion of fatal buprenorphine poisonings was similar in the parenteral BNX group and the parenteral BPN group. This is an interesting finding since it suggests that the presence of the opioid antidote naloxone in the product did not protect abusers from fatal buprenorphine poisoning, even though naloxone alone is regularly used to reverse opioid poisoning and intravenous BNX 


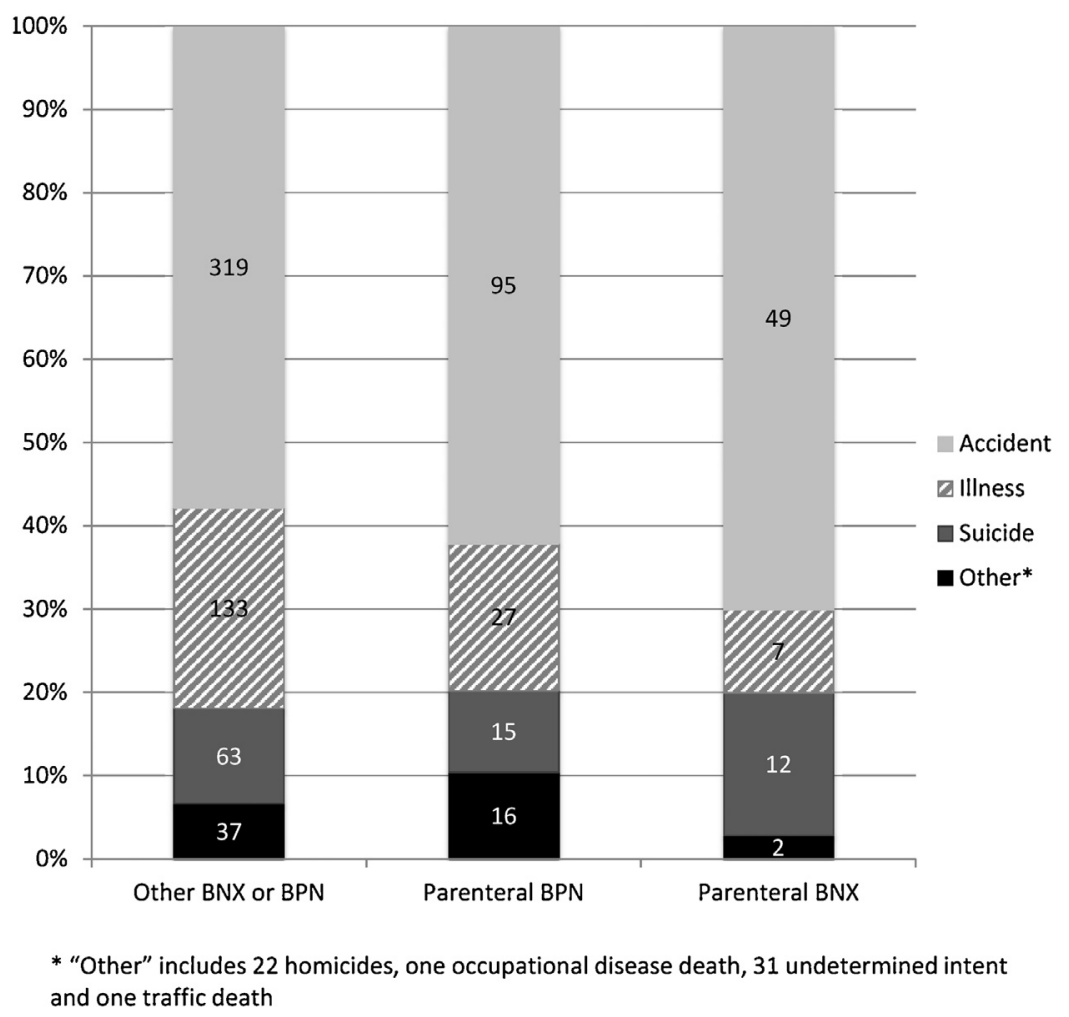

Fig. 3. Manner of death in each user group.

itself has been successfully used to reverse acute heroin poisoning [33]. Our previous study, in which the data set used was considerably smaller, found a significantly higher proportion of fatal buprenorphine poisonings in the parenteral BNX group compared to parenteral BPN. Although the current, larger data set did not confirm this finding the two studies are consistent in that neither shows evidence of a protective effect of naloxone. One reason for the lack of evidence on the protective effect may be the relatively short elimination half-life of naloxone (30-80 $\mathrm{min}$ ) when compared to that of buprenorphine (2-4h) [34]. This may lead to the protective effect being too short for the victim to survive.

The pharmacology of buprenorphine is complicated and not fully evaluated which makes understanding its abuse potential and toxicity difficult. In addition, its major active metabolite, norbuprenorphine, has a different pharmacological profile from buprenorphine. Norbuprenorphine is a potent, high affinity agonist at $\mu$, $\delta$, and $\kappa$ opioid receptors [35] whereas buprenorphine is a partial agonist at the $\mu$-opioid receptor with antagonist activity at the $\kappa$-opioid receptor. As concentrations and concentration ratios of buprenorphine and norbuprenorphine change over time due to metabolism and elimination there will be a changing pattern of pharmacological actions and interactions. Assigning toxicity to parent compound, metabolite or to relative concentrations is not possible with the data currently available. However, there is evidence that norbuprenorphine causes stronger respiratory depression than buprenorphine [15] and may contribute to the fatal outcome in poisoning cases

Studying the few fatal poisonings in our material in which there were no toxicological findings besides buprenorphine failed to reveal any novel patterns or risk factors for fatal poisoning. Based on background information, most of the victims of buprenorphine poisoning in our study had been abusing drugs for a long time. What caused them to die on that particular day, despite likely having developed some degree of opioid tolerance was unclear. Unfortunately, the background information in the forensic database is far from comprehensive and does not provide sufficient detail of the circumstances of these deaths to enable identification of important contributory factors.

Another complicating factor is that, the respiratory depression that can occur following the intake of buprenorphine, and in worst cases leads to death, can be a delayed process. As a result, buprenorphine levels at the time of death may have fallen below the laboratory cut-offs of some forensic laboratories [10].

In a recent Swedish study it was shown that the percentage of accidental drug poisonings was higher for those users positive for multiple psychoactive drugs in post-mortem toxicology compared to users positive for a single psychoactive drug [36]. Our results are well in line with this study in that the manner of death in the majority of the buprenorphine-positive cases was accident (60.0\% of all buprenorphine-positive cases, $61.8 \%$ of parenteral users), and most of the fatal poisonings were poly-drug poisonings (93.9\%). Poly-drug use is a common phenomenon in many countries [1,37]. Poly-drug use and the high prevalence of buprenorphine use among users of illegal drugs can create life-threatening interactions. Although some reports point out death cases in which nothing else was detected except buprenorphine [37] in most studies it has been shown that buprenorphine, when used alone, does not pose a great risk of intoxication but concomitant intake of other central nervous system depressants can induce severe respiratory depression and death [11].

Although parenteral use was common in our study material, the majority of the cases did not fulfil the urinary concentration criteria that would have clearly identified recent parenteral administration. An important limitation of the study is that the group "Other BNX and BPN", in addition to containing all those cases within the study material in which BNX and BPN had been administered sublingually as prescribed, is likely to include a number of parenteral users of both BPN and BNX. With the data available it was not possible to determine the relative proportions of sublingual vs. parenteral users in this group which may have 
affected the conclusions drawn from this material. In addition, the method used in this study to distinguish parenteral use of buprenorphine from sublingual use is only feasible in populations in which BNX is the sole or primary preparation used in OMT since medicinal use of BPN would further complicate the assessment.

The proportions of cases in each of the three groups were similar to those in our earlier study [27] and no significant changes in the number of cases in each subgroup or in the total number of cases were observed over the 5-year study period indicating that the route of administration and the relative frequency with which the different products were abused had not changed over that time period.

Individuals in the parenteral groups were in general younger than the others. Part of this difference may be explained by the group "other BNX or BPN" including elderly individuals taking prescribed buprenorphine preparations for the treatment of pain. It has been shown that with increasing age, prescribed use of opioid analgesics increases whereas non-prescribed use decreases [38]. The majority of the studied cases (64\%) were aged between 20 and 40 years and in this age group the percentage of parenteral users was much higher than in other age groups. In all three of the groups defined by urine drug levels, the age of the deceased increased over the five years of the study. This is in line with previous studies on fatal poisonings of drugs addicts in general where a similar trend has been observed [1]. A number of factors are likely to have contributed to this trend, including national efforts to treat those who abuse drugs. Treatment measures, including OMT, have certainly saved lives.

There has been very little heroin use in Finland in the past 10 years and opioid abuse in this country consists almost exclusively of buprenorphine use [1,39]. In Finland, buprenorphine has been one of the most important causes of fatal poisonings since the year 2000 - about half of the overall drug poisoning mortality (4.61 per 100,000 inhabitants aged between 15 and 64 in 2012) [1] is composed of buprenorphine poisoning. However, since the number of problem opioid users in Finland in 2012 was estimated to be about 13,800 whereas the number of patients in OMT in 2014 was 3000 [6], the majority of the users of buprenorphine are likely to be outside of OMT programs. Thus, despite the fatalities associated with abuse of buprenorphine, our results cannot be used to evaluate the safety of buprenorphine in OMT. In fact, previous studies have shown buprenorphine to be safer in OMT compared to methadone $[40,41]$.

Overall, the number of fatal drug poisonings has decreased in Finland in recent years. The same phenomenon has been seen in alcohol poisonings. In this study, the number of fatal buprenorphine poisonings decreased in the study period. The number of fatal poisonings would probably be higher if problem opioid users injected heroin instead of buprenorphine [42].

\section{Conclusion}

We conclude that parenteral use of buprenorphine-containing products is an ongoing and relatively prevalent phenomenon in Finland. In at least $28.8 \%$ of the buprenorphine-positive cases in the study period the drug had been administered parenterally, indicating abuse rather than medical use. The majority of the identified parenteral users had used BPN. Despite the presence of naloxone in BNX, this product is also abused and the percentage of parenteral BNX users in which the pathologist implicated buprenorphine in the cause of death was similar to that for parenteral BPN users. In most of the studied cases the deceased had used other drugs and/or alcohol along with buprenorphine which likely had contributed to the fatal outcome. The number of cases per year remained relatively constant over the study period but the mean age of the deceased increased significantly.

\section{Author contribution statement}

Ilkka Ojanperä conceived of the presented idea and encouraged Pirkko Kriikku to investigate the buprenorphine deaths in Finland. Pirkko Kriikku performed the database searches and statistical calculations, and wrote the manuscript with input from both Ilkka Ojanperä and Margareeta Häkkinen. Margareeta Häkkinen contributed with information from the clinical field and aided in interpreting the results. All authors discussed the results and contributed to the final manuscript.

\section{Declarations of interest}

None.

\section{Acknowledgements}

The authors would like to thank Dr Niall Doherty for his assistance. Parts of this study were presented at the annual meeting of the Nordic Association of Forensic Toxicologists (NAFT) in Trondheim, Norway in May 2017.

\section{References}

[1] K.W. Simonsen, H.M.E. Edvardsen, G. Thelander, I. Ojanperä, S. Thordardottir, L. V. Andersen, P. Kriikku, V. Vindenes, D. Christofferesen, G.J.M. Delaveris, J. Frost, Fatal poisoning in drug addicts in the Nordic countries in 2012, Forensic Sci. Int 248 (2015) 172-180.

[2] R.E. Johnson, E.C. Strain, L. Amass, Buprenorphine: how to use it right, Drug Alcohol Depend. 70 (2003) S59-S77.

[3] P. Hakkarainen, C. Tigerstedt, The breakthrough of Finnish substitution treatment (in Finnish), Yhteiskuntapolitiikka 70 (2005) 2 available at http:// www.julkari.fi/bitstream/handle/10024/101081/052hakkarainen.pdf?sequence $=1$. (Accessed 23 July 2018)

[4] H. Uosukainen, J. Kauhanen, S. Voutilainen, J. Föhr, M. Paasolainen, J. Tiihonen, K. Laitinen, I.N. Onyeka, J.S. Bell, Twelve-year trend in treatment seeking for buprenorphine abuse in Finland, Drug Alcohol Depend. 127 (2013) 207-214.

[5] K. Simojoki, H. Vorma, $\mathrm{H}$. Alho, A retrospective evaluation of patients switched from buprenorphine (Subutex) to the buprenorphine/naloxone combination (Suboxone), Subst. Abuse Treat. Prev. Policy 3 (2008) p16.

[6] EMCDDA country overview. Available at http://www.emcdda.europa.eu/ countries/finland\#. (Accessed 20 April 2017).

[7] S.L. Walsh, K.L. Preston, G.E. Bigelow, M.L. Stitzer, Acute administration of buprenorphine in humans: partial agonist and blockade effects, J. Pharmacol. Exp. Ther. 274 (1995) 361-372.

[8] A. Tracqui, P. Kintz, B. Ludes, Buprenorphine-related deaths among drug addicts in France: a report on 20 fatalities, J. Anal. Toxicol. 22 (1998) 430-434.

[9] M. Reynaud, G. Petit, D. Potard, P. Courty, Six deaths linked to concomitant use of buprenorphine and benzodiazepines, Addiction 93 (1998) 1385-1392.

[10] P. Kintz, Deaths involving buprenorphine: a compendium of French cases, Forensic Sci. Int. 121 (2001) 65-69.

[11] M. Häkkinen, T. Launiainen, E. Vuori, I. Ojanperä, Benzodiazepines and alcohol are associated with cases of fatal buprenorphine poisoning, Eur. J. Clin. Pharmacol. 68 (2012) 301-309.

[12] C. Cohier, L. Chevillard, S. Salle, P. Risède, O. Roussel, B. Mégarbane, Editor's highlight: neurorespiratory effects of buprenorphine and ethanol in combination: a mechanistic study of drug-drug interactions in the rat, Toxicol. Sci. 155 (2017) 389-399.

[13] B. Mégarbane, R. Hreiche, S. Pirnay, N. Marie, F.J. Baud, Does high-dose buprenorphine cause respiratory depression, Toxicol. Rev. 25 (2006) 79-85.

[14] C. Cohier, L. Chevillard, P. Risède, O. Roussel, B. Mégarbane, Respiratory effects of buprenorphine/naloxone alone and in combination with diazepam in naive and tolerant rats, Toxicol. Lett. 228 (2014) 75-84

[15] H. Alhaddad, S. Cisternino, X. Declèves, N. Tournier, J. Schlatter, F. Chiadmi, P. Risède, M. Smirnova, C. Besengez, J.M. Scherrmann, F.J. Baud, B. Mégarbane, Respiratory toxicity of buprenorphine results from the blockage of Pglycoprotein-mediated efflux of norbuprenorphine at the blood-brain barrier in mice, Crit. Care Med. 40 (2012) 3215-3223.

[16] H. Alhaddad, S. Cisternino, B. Saubamea, J. Schlatter, F. Chiadmi, P. Risède, M. Smirnova, V. Cochois-Guégan, N. Tournier, FJ. Baud, B. Mégarbane, Gender and strain contributions to the variability of buprenorphine-related respiratory toxicity in mice, Toxicology (2013) 99-108.

[17] S.W. Borron, C. Monier, P. Risede, F.J. Baud, Flunitrazepam variably alters morphine, buprenorphine, and methadone lethality in the rat, Human Exp. Toxicol. (2002) 599-605.

[18] G. Vidal-Trecan, I. Varescon, N. Nabet, A. Boissonnas, Intravenous use of prescribed sublingual buprenorphine tablets by drug users receiving maintenance therapy in France, Drug Alcohol. Depend. 69 (2013) 175-181. 
[19] M. Auriacombe, M. Fatséas, J. Dubernet, J.P. Daulouède, J. Tignol, French field experience with buprenorphine, Am. J. Addict. 13 (2004) S17-S28.

[20] R.A. Jenkinson, N.C. Clark, C.L. Fry, M. Dobbin, Buprenorphine diversion and injection in Melbourne, Australia: an emerging issue? Addiction 100 (2005) 197-205.

[21] H. Alho, D. Sinclair, E. Vuori, A. Holopainen, Abuse liability of buprenorphinenaloxone tablets in untreated IV drug users, Drug Alcohol Depend. 88 (2007) 75-78.

[22] C.K. Aitken, P.G. Higgs, M.E. Hellard, Buprenorphine injection in Melbourne, Australia: an update, Drug Alcohol Rev. 27 (2008) 197-199.

[23] B. Larance, N. Lintzeris, R. Ali, P. Dietze, R. Mattick, R. Jenkinson, N. White, L. Degenhard, The diversion and injection of a buprenorphine-naloxone soluble film formulation, Drug Alcohol Depend. 136 (2014) 21-27.

[24] B.N. Wikner, I. Öhman, T. Seldén, H. Druid, L. Brandt, H. Kieler, Opioid-related mortality and filled prescriptions for buprenorphine and methadone, Drug Alcohol Rev. 33 (2014) 491-498.

[25] B. Johnson, T. Richert, Diversion of methadone and buprenorphine by patients in opioid substitution treatment in Sweden: prevalence estimates and risk factors, int. J. Drug Policy 26 (2015) 183-190.

[26] E. Launonen, H. Alho, E. Kotovirta, I. Wallace, K. Simojoki, Diversion of opioid maintenance treatment medications and predictors for diversion among Finnish maintenance treatment patients, Int. J. Drug Policy 26 (2015) 875-882.

[27] M. Häkkinen, P. Heikman, I. Ojanperä, Parenteral buprenorphine-naloxone abuse is a major cause of fatal buprenorphine-related poisoning, Forensic Sci. Int. 23 (2013) 11-15.

[28] M. Sundström, A. Pelander, V. Angerer, M. Hutter, S. Kneisel, I. Ojanperä, A high sensitivity ultra-high performance liquid chromatography/high-resolution time-of-flight mass spectrometry (UHPLC-HR-TOFMS) method for screening synthetic cannabinoids and other drugs of abuse in urine, Anal. Bioanal. Chem. 405 (2013) 8463-8474.

[29] M.Sundström, A. Pelander, K. Simojoki, I. Ojanperä, Patterns of drug abuse among drug users with regular and irregular attendance for treatment as detected by comprehensive UHPLC-HR-TOF-MS, Drug Test. Anal. 8 (2016) 39-45.

[30] I. Rasanen, I. Kontinen, J. Nokua, I. Ojanperä, E. Vuori, Precise gas chromatography with retention time locking in comprehensive toxicological screening for drugs in blood, J. Chromatogr. B 788 (2003) 243-250.
[31] P. Heikman, M. Häkkinen, M. Gergov, I. Ojanperä, Urine naloxone concentration at different phases of buprenorphine maintenance treatment, Drug Test. Anal. 6 (2014) 220-225.

[32] E. Launonen, I. Wallace, E. Kotovirta, H. Alho, K. Simojoki, Factors associated with non-adherence and misuse of opioid maintenance treatment medications and intoxicating drugs among Finnish maintenance treatment patients, Drug Alcohol Depend. 162 (2016) 227-235.

[33] M.A. Yokell, N.D. Zaller, T.C. Green, M. McKenzie, J.D. Rich, Intravenous use of illicit buprenorphine/naloxone to reverse an acute heroin overdose, J. Opioid Manag. 8 (2012) 63.

[34] R.C. Baselt, Disposition of Toxic Drugs and Chemicals in Man, 11th ed., Biomedical Publications, Seal Beach, CA, USA, 2017.

[35] P. Huang, G.H. Kehner, A. Cowan, L.Y. Liu-Chen, Comparison of pharmacological activities of buprenorphine and norbuprenorphine: norbuprenorphine is a potent opioid agonist, J. Pharmacol. Exp. Ther. 297 (2001) 688-695.

[36] J. Ahlner, A. Holmgren, A.W. Jones, Demographics and post-mortem toxicology findings in deaths among people arrested multiple times for use of illicit drugs and/or impaired driving, Forensic Sci. Int. 265 (2016) 138-143.

[37] T. Seldén, J. Ahlner, H. Druid, R. Kronstrand, Toxicological and pathological findings in a series of buprenorphine related deaths. Possible risk factors for fatal outcome, Forensic Sci. Int. 220 (2012) 284-290.

[38] R. Paulose-Ram, R. Hirsch, C. Dillon, K. Losonczy, M. Cooper, Y. Ostchega Prescription and non-prescription analgesic use among the US adult population: results from the third National Health and Nutrition Examination Survey (NHANES III), Pharmacoepidemiol. Drug Saf. 12 (2003) 315-326.

[39] M. Häkkinen, T. Launiainen, E. Vuori, I. Ojanperä, Comparison of fatal poisonings by prescription opioids, Forensic Sci. Int. 222 (2012) 327-331.

[40] D. Marteau, R. McDonald, K. Patel, The relative risk of fatal poisoning by methadone or buprenorphine within the wider population of England and Wales, BMJ Open 5 (2015) 007629.

[41] D. Paone, E. Tuazon, M. Stajic, B. Sampson, B. Allen, H. Mantha, S. Kunins, Buprenorphine infrequently found in fatal overdose in New York City, Drug Alcohol Depend. 155 (2015) 298-301.

[42] J. Selin, R. Perälä, K. Stenius, A. Partanen, P. Rosenqvist, H. Alho, Opioid substitution treatment in Finland and other Nordic countries: Established treatment, varying practices, Nord. Stud. Alcohol Drugs 32 (2015) 311-324. 